\title{
TRANS-PLANCKIAN PHYSICS AND INFLATIONARY COSMOLOGY
}

\author{
ROBERT H. BRANDENBERGER \\ Physics Dept., Brown University, \\ Providence, R.I. 02912, USA \\ E-mail: rhb@het.brown.edu
}

\begin{abstract}
Due to the quasi-exponential redshifting which occurs during an inflationary period in the very early Universe, wavelengths which at the present time correspond to cosmological lengths are in general sub-Planckian during the early stages of inflation. This talk discusses two approaches to addressing this issue which both indicate that the standard predictions of inflationary cosmology - made using classical general relativity and weakly coupled scalar matter field theory - are not robust against changes in the physics on trans-Planckian scales. One approach makes use of modified dispersion relations for a usual free field scalar matter theory, the other uses some properties of space-time noncommutativity - a feature expected in string theory. Thus, it is possible that cosmological observations may be used as a window to explore trans-Planckian physics. We also speculate on a novel way of obtaining inflation based on modified dispersion relations for ordinary radiation.
\end{abstract}

\section{Introduction}

Inflationary cosmology ${ }^{1}$ is an elegant paradigm of the very early Universe which solves several conceptual problems of standard cosmology and leads to a predictive theory of the origin of cosmological fluctuations. The basic idea of inflation is to replace the time line of Standard Big Bang (SBB) cosmology (a late time phase of matter domination preceded by a period in which radiation is dominant and which begins with a cosmological singularity) by a modified time line for which during some time interval $I=\left[t_{i}, t_{R}\right]$ - long before the time of nucleosynthesis - the Universe is accelerating, often involving quasi-exponential expansion of the scale factor $a(t)$ (to set our notation, we will be writing the space-time metric for our homogeneous and isotropic background cosmology in the form

$$
d s^{2}=d t^{2}-a(t)^{2}\left[d x^{2}+d y^{2}+d z^{2}\right],
$$

where $t$ denotes physical time and $x, y, z$ are the three Euclidean comoving spatial coordinates - we are neglecting the spatial curvature). The time $t_{i}$ 
stands for the beginning of the inflationary phase, the time $t_{R}$ stands for the end, the time of "reheating".

Inflationary cosmology solves several of the conceptual problems of SBB cosmology. In particular, it resolves the homogeneity problem, the inability of SBB cosmology to address the reason for the near isotropy of the cosmic microwave background (CMB), it explains the spatial flatness of the Universe, and it provides the first ever mechanism of cosmological structure formation based on causal physics.

Let us briefly recall how inflationary cosmology leads to the existence of a mechanism for using causal microphysics for producing fluctuations on cosmological scales (when measured today), which at the time of equal matter and radiation had a physical wavelength larger than the Hubble radius $l_{H}(t)=H^{-1}(t)$, where $H=\dot{a} / a$ is the expansion rate (for references to the original literature see textbook treatments ${ }^{2,3}$ ). The relevant spacetime sketch is shown in Fig. 1. If we trace a fixed comoving length scale (corresponding e.g. to CMB anisotropies on a fixed large angular scale) back into the past, then in SBB cosmology this scale is larger than the horizon (which in standard cosmology is $H^{-1}$ - up to a numerical coefficient of order one) at early times. The time when such scales "cross" the horizon is in fact later than $t_{\text {rec. }}$. Thus, it appears impossible to explain the origin of the primordial fluctuations measured in the $\mathrm{CMB}$ without violating causality a.

In an inflationary Universe (taking exponential inflation to be specific), during the period of exponential expansion the physical wavelength corresponding to a fixed comoving scale decreases exponentially as we go back in time, whereas the Hubble radius $l_{H}(t)$ is constant ${ }^{\mathrm{b}}$. Thus, as long as the period of inflation is sufficiently long, all scales of cosmological interest today originate inside the Hubble radius in the early stages of inflation. As will be shown in the next section, the squeezing of quantum vacuum fluctuations when they exit and propagate outside the Hubble radius is the mechanism for the origin of fluctuations in inflationary cosmology.

The same background dynamics which yields the causal generation mechanism for cosmological fluctuations, the most spectacular success of inflationary cosmology, bears in it the nucleus of the trans-Planckian problem. This can also be seen from Fig. 1. If inflation lasts only slightly longer than the minimal time it needs to last in order to solve the horizon problem

aTopological defect models are a counterexample to this "standard dogma"

${ }^{\mathrm{b}}$ The Hubble radius measures the distance over which microphysics can act coherently at a fixed time $t$, and in inflationary cosmology differs in a crucial way from the causal horizon (the forward light cone, which becomes exponentially larger). 


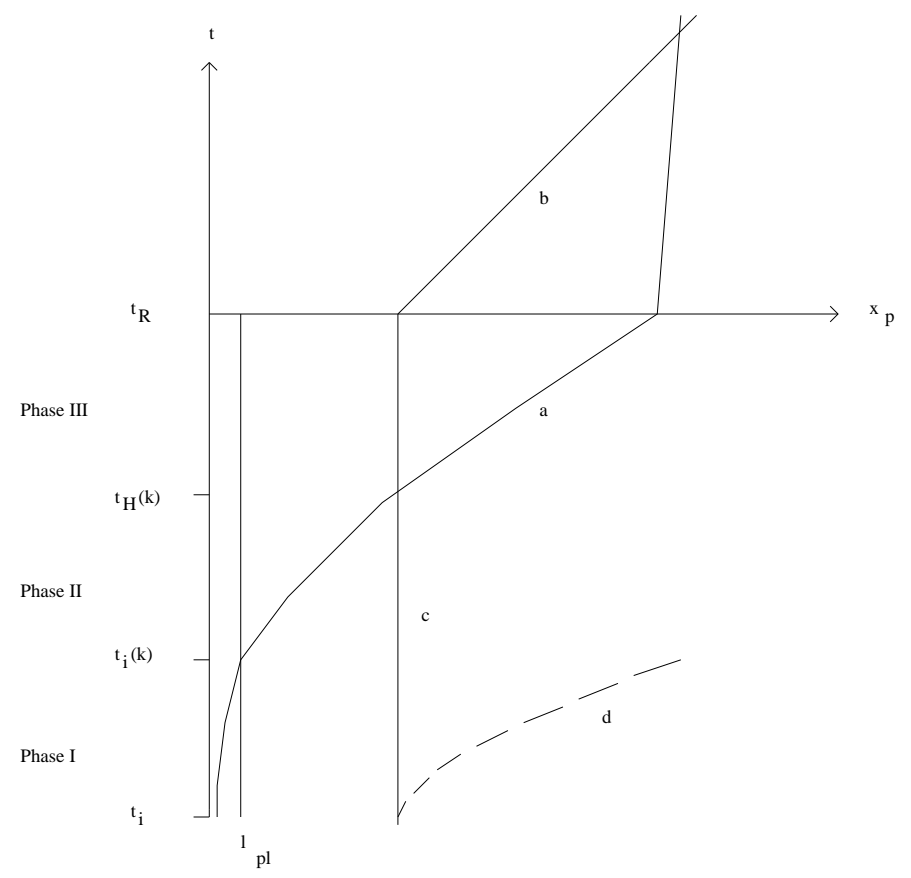

Figure 1. Space-time diagram (physical distance vs. time) showing how inflationary cosmology provides a causal mechanism for producing cosmological fluctuations. The line labeled a) is the physical wavelength associated with a fixed comoving scale $k$. The line $\mathrm{b}$ ) is the Hubble radius or horizon in SBB cosmology. Note that at $t_{\text {rec }}$ the fluctuation is outside the Hubble radius. Curve c) shows the Hubble radius during inflation. As depicted, at early times during inflation the curve a) is inside the Hubble radius, thus allowing for a causal generation mechanism for fluctuations on the corresponding scale. The horizon in inflationary cosmology is shown in curve d). The graph also demonstrates the trans-Planckian problem of inflationary cosmology: at very early times, the wavelength is smaller than the Planck scale $\ell_{\mathrm{Pl}}$ (Phase $\mathrm{I}$ ), at intermediate times it is larger than $\ell_{\mathrm{Pl}}$ but smaller than the Hubble radius $H^{-1}$ (Phase II), and at late times during inflation it is larger than the Hubble radius (Phase III).

and to provide a causal generation mechanism for CMB fluctuations, then the corresponding physical wavelength of these fluctuations is smaller than the Planck length at the beginning of the period of inflation. The theory of cosmological perturbations is based on classical general relativity coupled to a weakly coupled scalar field description of matter. Both the theory of gravity and of matter will break down on trans-Planckian scales, and this immediately leads to the trans-Planckian problem: are the predictions of standard inflationary cosmology robust against effects of trans-Planckian physics ${ }^{4}$ ? To answer this question, we need to give the reader a short review of the standard theory of cosmological fluctuations. 


\section{Fluctuations in Inflationary Cosmology}

In the following we give an overview of the quantum theory of cosmological perturbations (see review articles ${ }^{5}$ for details and references to the original literature). Since gravity is a purely attractive force, and since the fluctuations on scales of the CMB anisotropies were small in amplitude when the anisotropies were generated, the fluctuations had to have been very small in the early Universe. Thus, a linearized analysis of the fluctuations is justified. In this case, the Fourier modes of the cosmological fluctuations evolve independently.

The basic idea of the theory of cosmological perturbations is to quantize the linear fluctuations about a classical background cosmology. The starting point is the full action of gravity plus matter

$$
S=\int \mathrm{d}^{4} x \sqrt{-g} R+S_{\mathrm{m}},
$$

where the first term is the usual Einstein-Hilbert action for gravity, $R$ being the Ricci scalar and $g$ the determinant of the metric, and $S_{\mathrm{m}}$ is the matter action. For the sake of simplicity, and since it is the usual assumption in simple inflationary Universe models, we take matter to be described by a single minimally coupled scalar field $\varphi$. Then, we separate the metric and matter into classical background variables $g_{\mu \nu}^{(0)}, \varphi^{(0)}$ which depend only on time, and fluctuating fields $\delta g_{\mu \nu}, \delta \varphi$ which depend on space and time and have vanishing spatial average:

$$
g_{\mu \nu}=g_{\mu \nu}^{(0)}(\eta)+\delta g_{\mu \nu}(\eta, \mathbf{x}), \quad \varphi=\varphi^{(0)}(\eta)+\delta \varphi(\eta, \mathbf{x}) .
$$

We will focus on scalar metric fluctuations, the fluctuating degrees of freedom which couple to matter perturbations ${ }^{\mathrm{c}}$.

The description of scalar metric perturbations is complicated by the fact that some perturbation modes correspond to space-time reparametrizations of a homogeneous and isotropic cosmology. A simple way to address this issue of gauge is to work in a system of coordinates which completely fixes the gauge. A simple choice is the longitudinal gauge, in which the metric takes the form ${ }^{5}$

$$
\mathrm{d} s^{2}=a^{2}(\eta)\left[(1+2 \Phi) d \eta^{2}-(1-2 \Psi) \delta_{i j} \mathrm{~d} x^{i} \mathrm{~d} x^{j}\right],
$$

where the space- and time-dependent functions $\Phi$ and $\Psi$ are the two physical metric degrees of freedom which describe scalar metric fluctuations, and $\eta$ is conformal time (related to physical time $t$ via $d t=a(\eta) d \eta$ ). The

${ }^{c}$ Greek variables run over space-time indices, Latin variables only over spatial indices. 
fluctuations of matter fields give additional degrees of freedom for scalar metric fluctuations. In the simple case of a single scalar matter field, the matter field fluctuation is $\delta \varphi$.

In the absence of anisotropic stress, it follows from the $i \neq j$ Einstein equations that the two metric fluctuation variables $\Phi$ and $\Psi$ coincide. Due to the Einstein constraint equation, the remaining metric fluctuation $\Psi$ is determined by the matter fluctuation $\delta \varphi$. It is clear from this analysis of the physical degrees of freedom that the action for scalar metric fluctuations must be expressible in terms of the action of a single free scalar field $v$ with a time dependent mass (determined by the background cosmology). As shown in ${ }^{6}$ (see also ${ }^{7}$ ), this field is

$$
v=a\left(\delta \varphi+\frac{\varphi^{(0)^{\prime}}}{\mathcal{H}} \Psi\right)=z \mathcal{R},
$$

where $\mathcal{H}=a^{\prime} / a$ (a prime denoting the derivative with respect to $\eta$ ),

$$
z \equiv a \frac{\varphi^{(0)^{\prime}}}{\mathcal{H}},
$$

and $\mathcal{R}$ denotes the curvature perturbation in comoving gauge ${ }^{8}$. The action for scalar metric fluctuations is ${ }^{9}$

$$
\delta S_{\mathcal{R}}=\frac{1}{2} \int \mathrm{d}^{4} \mathbf{x}\left[\left(v_{\mathbf{k}}^{\prime}\right)^{2}-\delta^{i j} v_{\mathbf{k}, i} v_{\mathbf{k}, j}+\frac{z^{\prime \prime}}{z} v_{\mathbf{k}}^{2}\right],
$$

which leads to the equation of motion

$$
v_{\mathbf{k}}^{\prime \prime}+\left(k^{2}-\frac{z^{\prime \prime}}{z}\right) v_{\mathbf{k}}=0
$$

the equation of motion of a harmonic oscillator with time-dependent mass, the time dependence being given by $z(\eta)$ which is a function of the background cosmology. Note that if $a(\eta)$ is a power of $\eta$, then $\varphi_{0}^{\prime}$ and $\mathcal{H}$ scale with the same power of $\eta$, and the variable $z$ is then proportional to $a$.

At this point we can summarize the mechanism by which cosmological fluctuations are generated in inflationary cosmology: we canonically quantize the linearized metric/matter fluctuations about a classical background cosmology, solve the resulting dynamical problem as a standard initial value problem, setting off all Fourier modes of the scalar field $v$ in their vacuum state at the initial time (e.g. the beginning of the period of inflation).

The equation (8) is a harmonic oscillator equation with a timedependent mass given by $z^{\prime \prime} / z$. On scales smaller than the Hubble radius $\left(t<t_{\mathrm{H}}(k)\right)$, the mass term is negligible, and the mode functions oscillate with constant amplitude. On scales larger than the Hubble radius, however, the mass term dominates and the $k^{2}$ term can be neglected. The mode 
functions no longer oscillate. In an expanding background, the dominant mode of $v_{\mathbf{k}}(\eta)$ scales as $z(\eta)$. Note that it is incorrect to assume that fluctuations are created at the time of Hubble radius crossing (an impression one could get from reading $\left.{ }^{10}\right)$. Rather, the role of the time $t_{\mathrm{H}}(k)$ of Hubble radius crossing is to set the time when the classical mode functions cease to oscillate and begin to increase in amplitude (squeezing).

The quantum mechanical interpretation of the two phases $t<t_{\mathrm{H}}(k)$ and $t>t_{\mathrm{H}}(k)$ is the following: on sub-Hubble scales we have oscillating quantum vacuum fluctuations and there is no particle production. Once the scales cross the Hubble radius, the mode functions begin to grow and the fluctuations get frozen. The initial vacuum state then becomes highly squeezed for $t \gg t_{\mathrm{H}}(k) \mathrm{d}$. The squeezing leads to the generation of effectively classical cosmological perturbations.

For cosmological applications, it is particularly interesting to calculate the power spectrum of the curvature perturbation $\mathcal{R}$, defined as

$$
\mathcal{P}_{\mathcal{R}}(k)=\frac{k^{3}}{2 \pi^{2} z^{2}}\left|v_{k}\right|^{2} .
$$

This last quantity can be estimated very easily. From the fact that on scales larger than the Hubble radius the mode functions are proportional to $a(\eta)$, we find

$$
\mathcal{P}_{\mathcal{R}}(k) \sim \frac{k^{3}}{2 \pi^{2}} \frac{1}{2 k} \frac{1}{a^{2}\left[\eta_{\mathrm{H}}(k)\right]},
$$

where $\eta_{\mathrm{H}}(k)$ is the conformal time of Hubble radius crossing for the mode with comoving wavenumber $k$. Note that the second factor on the r.h.s. of (10) represents the vacuum normalization of the wave function.

As is evident from Figure 1, the standard theory of cosmological fluctuations summarized in this section relies on extrapolating classical general relativity and weakly coupled scalar matter field theory to length scales smaller than the Planck length. Thus, it is legitimate to ask whether the predictions resulting from this theory are sensitive to modifications of physics on physical length scales smaller than the Planck length. There are various ways in which such physics could lead to deviations from the standard predictions. First, new physics could lead to a non-standard evolution of the initial vacuum state of fluctuations in Period I, such that at Hubble radius crossing the state is different from the vacuum state. A

\footnotetext{
d The equation of motion for gravitational waves in an expanding background cosmology is identical to (8) with $z(\eta)$ replaced by $a(\eta)$, and thus the physics is identical. The case of gravitational waves was first discussed in ${ }^{11}$.
} 
model of realizing this scenario is summarized in the following section. Secondly, trans-Planckian physics may lead to different boundary conditions, thus resulting in a different final state. A string-motivated example for this scenario is given in Section 4.

\section{Trans-Planckian Analysis I: Modified Dispersion Relations}

The simplest way of modeling the possible effects of trans-Planckian physics, while keeping the mathematical analysis simple, is to replace the linear dispersion relation $\omega_{\text {phys }}=k_{\text {phys }}$ of the usual equation for cosmological perturbations by a non standard dispersion relation $\omega_{\text {phys }}=\omega_{\text {phys }}(k)$ which differs from the standard one only for physical wavenumbers larger than the Planck scale. This method was introduced ${ }^{12,13}$ in the context of studying the dependence of thermal spectrum of black hole radiation on trans-Planckian physics. In the context of cosmology, it has been shown $14,15,16$ that this amounts to replacing $k^{2}$ appearing in (8) with $k_{\text {eff }}^{2}(n, \eta)$ defined by

$$
k^{2} \rightarrow k_{\mathrm{eff}}^{2}(k, \eta) \equiv a^{2}(\eta) \omega_{\mathrm{phys}}^{2}\left[\frac{k}{a(\eta)}\right] .
$$

For a fixed comoving mode, this implies that the dispersion relation becomes time-dependent. Therefore, the equation of motion of the quantity $v_{k}(\eta)$ takes the form

$$
v_{k}^{\prime \prime}+\left[k_{\text {eff }}^{2}(k, \eta)-\frac{a^{\prime \prime}}{a}\right] v_{k}=0 .
$$

A more rigorous derivation of this equation, based on a variational principle, has been provided ${ }^{17}$ (see also Ref. ${ }^{18}$ ).

The evolution of modes thus must be considered separately in three phases, see Fig. 1. In Phase I the wavelength is smaller than the Planck scale, and trans-Planckian physics can play an important role. In Phase II, the wavelength is larger than the Planck scale but smaller than the Hubble radius. In this phase, trans-Planckian physics will have a negligible effect (this statement can be quantified ${ }^{10}$ ). Hence, by the analysis of the previous section, the wave function of fluctuations is oscillating in this phase,

$$
v_{k}=B_{1} \exp (-i k \eta)+B_{2} \exp (i k \eta)
$$

with constant coefficients $B_{1}$ and $B_{2}$. In the standard approach, the initial conditions are fixed in this region and the usual choice of the vacuum state leads to $B_{1}=1 / \sqrt{2 k}, B_{2}=0$. Phase III starts at the time $t_{\mathrm{H}}(k)$ when the 
mode crosses the Hubble radius. During this phase, the wave function is squeezed.

One source of trans-Planckian effects ${ }^{14,15}$ on observations is the possible non-adiabatic evolution of the wave function during Phase I. If this occurs, then it is possible that the wave function of the fluctuation mode is not in its vacuum state when it enters Phase II and, as a consequence, the coefficients $B_{1}$ and $B_{2}$ are no longer given by the standard expressions above. In this case, the wave function will not be in its vacuum state when it crosses the Hubble radius, and the final spectrum will be different. In general, $B_{1}$ and $B_{2}$ are determined by the matching conditions between Phase I and II. By focusing only ${ }^{10}$ on trans-Planckian effects on the local vacuum wave function at the time $t_{\mathrm{H}}(k)$, one misses this important potential source of trans-Planckian signals in the CMB. If the dynamics is adiabatic throughout (in particular if the $a^{\prime \prime} / a$ term is negligible), the WKB approximation holds and the solution is always given by

$$
v_{k}(\eta) \simeq \frac{1}{\sqrt{2 k_{\mathrm{eff}}(k, \eta)}} \exp \left[-i \int_{\eta_{\mathrm{i}}}^{\eta} k_{\mathrm{eff}} \mathrm{d} \tau\right],
$$

where $\eta_{i}$ is some initial time. Therefore, if we start with a positive frequency solution only and use this solution, we find that no negative frequency solution appears. Deep in Region II where $k_{\text {eff }} \simeq k$ the solution becomes

$$
v_{k}(\eta) \simeq \frac{1}{\sqrt{2 k}} \exp (-i \phi-i k \eta)
$$

i.e. the standard vacuum solution times a phase which will disappear when we calculate the modulus. To obtain a modification of the inflationary spectrum, it is sufficient to find a dispersion relation such that the WKB approximation breaks down in Phase I.

A concrete class of dispersion relations for which the WKB approximation breaks down is

$$
k_{\text {eff }}^{2}(k, \eta)=k^{2}-k^{2}\left|b_{m}\right|\left[\frac{\ell_{\mathrm{pl}}}{\lambda(\eta)}\right]^{2 m},
$$

where $\lambda(\eta)=2 \pi a(\eta) / k$ is the wavelength of a mode. If we follow the evolution of the modes in Phases I, II and III, matching the mode functions and their derivatives at the junction times, the calculation ${ }^{14,15,19}$ demonstrates that the final spectral index is modified and that superimposed oscillations appear.

However, the above example suffers from several problems. First, in inflationary models with a long period of inflationary expansion, the dispersion relation (16) leads to complex frequencies at the beginning of inflation for scales which are of current interest in cosmology. Furthermore, the 
initial conditions for the Fourier modes of the fluctuation field have to be set in a region where the evolution is non-adiabatic and the use of the usual vacuum prescription can be questioned. These problems can be avoided in a toy model in which we follow the evolution of fluctuations in a bouncing cosmological background which is asymptotically flat in the past and in the future. The analysis ${ }^{20}$ shows that even in this case the final spectrum of fluctuations depends on the specific dispersion relation used.

An example of a dispersion relation which breaks the WKB approximation in the trans-Planckian regime but does not lead to the problems mentioned in the previous paragraph was investigated in ${ }^{17}$. It is a dispersion relation which is linear for both small and large wavenumbers, but has an intermediate interval during which the frequency decreases as the wavenumber increases, much like what happens in (16). The violation of the WKB condition occurs for wavenumbers near the local minimum of the $\omega(k)$ curve.

\section{Trans-Planckian Analysis II: Space-Time Uncertainty Relation}

A justified criticism against the method summarized in the previous section is that the non-standard dispersion relations used are completely ad hoc, without a clear basis in trans-Planckian physics. There has been a lot of recent work $21,22,23,24$ on the implication of space-space uncertainty relations 25,26 on the evolution of fluctuations. The application of the uncertainty relations on the fluctuations lead to two effects ${ }^{27,28}$. Firstly, the equation of motion of the fluctuations in modified. Secondly, for fixed comoving length scale $k$, the uncertainty relation is saturated before a critical time $t_{i}(k)$. Thus, in addition to a modification of the evolution, trans-Planckian physics leads to a modification of the boundary condition for the fluctuation modes. The upshot of this work is that the spectrum of fluctuations is modified.

We have recently studied ${ }^{29}$ the implications of the stringy space-time uncertainty relation 30,31

$$
\Delta x_{\text {phys }} \Delta t \geq l_{s}^{2}
$$

on the spectrum of cosmological fluctuations. Again, application of this uncertainty relation to the fluctuations leads to two effects. Firstly, the coupling between the background and the fluctuations is nonlocal in time, thus leading to a modified dynamical equation of motion. Secondly, the uncertainty relation is saturated at the time $t_{i}(k)$ when the physical wavelength equals the string scale $l_{s}$. Before that time it does not make sense 
to talk about fluctuations on that scale. By continuity, it makes sense to assume that fluctuations on scale $k$ are created at time $t_{i}(k)$ in the local vacuum state (the instantaneous WKB vacuum state).

Let us for the moment neglect the nonlocal coupling between background and fluctuation, and thus consider the usual equation of motion for fluctuations in an accelerating background cosmology. We distinguish two ranges of scales. Ultraviolet modes are generated at late times when the Hubble radius is larger than $l_{s}$. On these scales, the spectrum of fluctuations does not differ from what is predicted by the standard theory, since at the time of Hubble radius crossing the fluctuation mode will be in its vacuum state. However, the evolution of infrared modes which are created when the Hubble radius is smaller than $l_{s}$ is different. The fluctuations undergo less squeezing than they do in the absence of the uncertainty relation, and hence the final amplitude of fluctuations is lower. From the equation (10) for the power spectrum of fluctuations, and making use of the condition

$$
a\left(t_{i}(k)\right)=k l_{s}
$$

for the time $t_{i}(k)$ when the mode is generated, it follows immediately that the power spectrum is scale-invariant

$$
\mathcal{P}_{\mathcal{R}}(k) \sim k^{0} .
$$

In the standard scenario of power-law inflation the spectrum is red $\left(\mathcal{P}_{\mathcal{R}}(k) \sim k^{n-1}\right.$ with $\left.n<1\right)$. Taking into account the effects of the nonlocal coupling between background and fluctuation mode leads ${ }^{29}$ to a modification of this result: the spectrum of fluctuations in a power-law inflationary background is in fact blue $(n>1)$.

Note that, if we neglect the nonlocal coupling between background and fluctuation mode, the result of (19) also holds in a cosmological background which is NOT accelerating. Thus, we have a method of obtaining a scaleinvariant spectrum of fluctuations without inflation. This result has also been obtained in ${ }^{32}$, however without a microphysical basis for the prescription for the initial conditions.

\section{Non-Commutative Inflation}

A key problem with the method of modified dispersion relations is the issue of back-reaction 33,34 . If the mode occupation numbers of the fluctuations at Hubble radius crossing are significant, the danger arises that the backreaction of the fluctuations will in fact prevent inflation. Another constraint

arises from the observational limits on the flux of ultra-high-energy cosmic 
rays. Such cosmic rays would be produced ${ }^{35}$ in the present Universe if Trans-Planckian effects of the type discussed in the two previous sections were present. These issues are currently under investigation.

Surprisingly, it has been realized ${ }^{36}$ that back-reaction effects due to modified dispersion relations (which in turn are motivated by string theory) might it fact yield a method of obtaining inflation from pure radiation. In this section, $p$ and $\omega$ will denote physical wavenumber and frequency, respectively.

One of the key features expected from string theory is the existence of a minimal length, or equivalently a maximal wavenumber $p_{\max }$. Thus, if we consider the dispersion relation for pure radiation in string theory, the dispersion relation must saturate or turn over at $p_{\max }$. Some cosmological consequences of a dispersion relation which saturates at $p_{\max }$, i.e. for which the frequency $\omega$ diverges as $k \rightarrow p_{\max }$ were explored recently ${ }^{38}$. It was found that a realization of the varying speed of light scenario can be obtained.

Recent work ${ }^{36}$ has focused on the case when the dispersion relation turns over at $p_{\max }$, i.e. if the frequency is increased, then before $p(\omega)$ reaches $p_{\max }$, the wavenumber begins to decrease again. This implies that for each value of $p$ there are two states corresponding to two different frequencies, i.e. that there are two branches of the dispersion relation. A class of such dispersion relations is given by

$$
\omega^{2}-p^{2} f^{2}=0, f(\omega)=1+(\lambda E)^{\alpha},
$$

where $\alpha$ is a free parameter.

Let us now consider an expanding Universe with such a dispersion relation, and assume that in the initial state both branches are populated up to a frequency much larger than the frequency at which the dispersion relation turns over. As the Universe expands, the physical wavenumber of all modes decreases. However, this implies - in contrast to the usual situation - that the energy of the upper branch states increases. This is what one wants to be able to achieve an inflationary cosmology. Eventually, the high energy states will decay into the lower branch states (which have the usual equation of state of radiation), thus leading to a graceful exit from inflation.

To check whether the above heuristic scenario is indeed realized, one must compute the equation of state corresponding to the dispersion relation (20). The spectrum is deformed, and a thermodynamic calculation ${ }^{36}$ yields an equation of state which in the high density limit tends to $P=1 /[3(1-$ $\alpha)] \rho$ where $P$ stands for the pressure. Thus, we see that there is a narrow 
range of values of $\alpha$ which indeed give the correct equation of state for power-law inflation.

In such an inflationary scenario, the fluctuations are of thermal origin. Taking the initial r.m.s. amplitude of the mass fluctuations on thermal length scale $T^{-1}$ to be order unity, assuming random superposition of such fluctuations on larger scales to compute the amplitude of fluctuations when a particular comoving length scale exits the Hubble radius, and using the usual theory of cosmological fluctuations to track the fluctuations to the time when the scale enters the Hubble radius, one finds a spectrum of fluctuations with the same slope as in regular power-law inflation, and with an amplitude which agrees with the value required from observations if the fundamental length scale $l_{s}$ at which the dispersion relation turns over is about an order of magnitude larger than the Planck length.

\section{Conclusions}

Due to the exponential redshifting of wavelengths, present cosmological scales originate at wavelengths smaller than the Planck length early on during the period of inflation. Thus, Planck physics may well encode information in these modes which can now be observed in the spectrum of microwave anisotropies. Two examples have been shown to demonstrate the existence of this "window of opportunity" to probe trans-Planckian physics in cosmological observations. The first method makes use of modified dispersion relations to probe the robustness of the predictions of inflationary cosmology, the second applies the stringy space-time uncertainty relation on the fluctuation modes. Both methods yield the result that trans-Planckian physics may lead to measurable effects in cosmological observables. An important issue which must be studied more carefully is the back-reaction of the cosmological fluctuations (see e.g. ${ }^{37}$ for a possible formalism). As demonstrated in the final section, it is possible that trans-Planckian physics can in fact lead to dramatic changes even in the background cosmology.

A final remark related to other work on the trans-Planckian problem for inflationary cosmology: in our view, nontrivial trans-Planckian physics (which cannot be described in any way by a free scalar field) produces excitations about the Bunch-Davies vacuum state for a finite range of wavelengths. On these wavelengths, the excited state may (but need not) look like a nontrivial $\alpha$ vacuum of a free scalar field theory in de Sitter space $39,40,41,42$. However, globally the state is NOT an $\alpha$ vacuum, so that the recent discussions about possible problems with $\alpha$ vacua ${ }^{43,44,45,46}$ are not crucial to the question of whether Planck-scale physics can lead to distinctive signatures observable in cosmology. 


\section{Acknowledgments}

This is a slightly updated version of the contribution to the proceedings of the CosPA2002 meeting at National Taiwan University held May 31 - June 2 2002. I am grateful to the organizers of CosPA2002 for their wonderful hospitality, and to S. Alexander, P.-M. Ho, S. Joras, J. Magueijo and J. Martin for collaboration. The research was supported in part by the U.S. Department of Energy under Contract DE-FG02-91ER40688, TASK A.

\section{References}

1. A. H. Guth, Phys. Rev. D23, 347 (1981).

2. A. Linde, "Particle Physics and Inflationary Cosmology", Harwood Academic, Chur, (1990).

3. A. Liddle and D. Lyth, "Cosmological inflation and large-scale structure, Cambridge Univ. Press, Cambridge, (2000).

4. R. H. Brandenberger, arXiv:hep-ph/9910410.

5. V. F. Mukhanov, H. A. Feldman and R. H. Brandenberger, Phys. Rept. 215, 203 (1992).

6. V. F. Mukhanov, JETP Lett. 41, 493 (1985) [Pisma Zh. Eksp. Teor. Fiz. 41, $402(1985)]$.

7. V. Lukash, Sov. Phys. JETP 52, 807 (1980).

8. D. H. Lyth, Phys. Rev. D31, 1792 (1985).

9. V. F. Mukhanov, Sov. Phys. JETP 67 (1988) 1297 [Zh. Eksp. Teor. Fiz. 94N7 (1988 ZETFA,94,1-11.1988) 1].

10. N. Kaloper, M. Kleban, A. E. Lawrence and S. Shenker, arXiv:hepth/0201158.

11. L. P. Grishchuk, Sov. Phys. JETP 40, 409 (1975) [Zh. Eksp. Teor. Fiz. 67, 825 (1974)].

12. W. G. Unruh, Phys. Rev. D51, 2827 (1995).

13. S. Corley and T. Jacobson, Phys. Rev. D54, 1568 (1996) [arXiv:hepth/9601073].

14. J. Martin and R. H. Brandenberger, Phys. Rev. D63, 123501 (2001) [arXiv:hep-th/0005209].

15. R. H. Brandenberger and J. Martin, Mod. Phys. Lett. A16, 999 (2001) [arXiv:astro-ph/0005432].

16. J. C. Niemeyer, Phys. Rev. D63, 123502 (2001) [arXiv:astro-ph/0005533].

17. M. Lemoine, M. Lubo, J. Martin and J. P. Uzan, Phys. Rev. D65, 023510 (2002) [arXiv:hep-th/0109128].

18. T. Jacobson and D. Mattingly, Phys. Rev. D63, 041502 (2001) [arXiv:hepth/0009052].

19. J. Martin and R. H. Brandenberger, Phys. Rev. D65, 103514 (2002) [arXiv:hep-th/0201189].

20. R. H. Brandenberger, S. E. Joras and J. Martin, arXiv:hep-th/0112122.

21. R. Easther, B. R. Greene, W. H. Kinney and G. Shiu, Phys. Rev. D64, 103502 (2001) [arXiv:hep-th/0104102]. 
22. A. Kempf and J. C. Niemeyer, Phys. Rev. D64, 103501 (2001) [arXiv:astro$\mathrm{ph} / 0103225]$.

23. R. Easther, B. R. Greene, W. H. Kinney and G. Shiu, arXiv:hep-th/0110226.

24. F. Lizzi, G. Mangano, G. Miele and M. Peloso, JHEP 0206, 049 (2002) [arXiv:hep-th/0203099].

25. D. Amati, M. Ciafaloni and G. Veneziano, Phys. Lett. B197, 81 (1987).

26. D. J. Gross and P. F. Mende, Nucl. Phys. B303, 407 (1988).

27. A. Kempf, Phys. Rev. D63, 083514 (2001) [arXiv:astro-ph/0009209].

28. S. F. Hassan and M. S. Sloth, arXiv:hep-th/0204110.

29. R. Brandenberger and P. M. Ho, Phys. Rev. D66, 023517 (2002) [arXiv:hepth/0203119].

30. T. Yoneya, Mod. Phys. Lett. A4, 1587 (1989).

31. M. Li and T. Yoneya, arXiv:hep-th/9806240.

32. S. Hollands and R. M. Wald, arXiv:gr-qc/0205058.

33. T. Tanaka, arXiv:astro-ph/0012431.

34. A. A. Starobinsky, Pisma Zh. Eksp. Teor. Fiz. 73, 415 (2001) [JETP Lett. 73, 371 (2001)] [arXiv:astro-ph/0104043].

35. A. A. Starobinsky and I. I. Tkachev, arXiv:astro-ph/0207572.

36. S. Alexander, R. Brandenberger and J. Magueijo, arXiv:hep-th/0108190.

37. L. R. Abramo, R. H. Brandenberger and V. F. Mukhanov, Phys. Rev. D56, 3248 (1997) [arXiv:gr-qc/9704037].

38. S. Alexander and J. Magueijo, arXiv:hep-th/0104093.

39. U. H. Danielsson, Phys. Rev. D 66, 023511 (2002) [arXiv:hep-th/0203198].

40. U. H. Danielsson, JHEP 0207, 040 (2002) [arXiv:hep-th/0205227].

41. R. Easther, B. R. Greene, W. H. Kinney and G. Shiu, Phys. Rev. D 66, 023518 (2002) [arXiv:hep-th/0204129].

42. K. Goldstein and D. A. Lowe, arXiv:hep-th/0208167.

43. T. Banks and L. Mannelli, arXiv:hep-th/0209113.

44. M. B. Einhorn and F. Larsen, arXiv:hep-th/0209159.

45. N. Kaloper, M. Kleban, A. Lawrence, S. Shenker and L. Susskind, arXiv:hepth/0209231.

46. U. H. Danielsson, arXiv:hep-th/0210058. 